Results Rapamycin made the expression and activation of KLF2 strongly reduce by $75.6 \%$ and $78.2 \%$ so as to induce long-term coronary endothelial dysfunction. In HUVECs, rapamycin made basal eNOS and t-PA decrease by $80 \%$ and $87.8 \%$, while making basal PAI-1 and TF increase by 2.5 and 1.5 -fold. After treatment by statins (especially lovastatin), the expression of KLF2 was increased by 3.8-fold nearly reversing to normal state.

Conclusions Taken together, these observations indicate that statindependent induction of KLF2 provides a new treatment for stent thrombosis induced by rapamycin releasing from drug-eluting stents.

\section{e0521 CLINICAL EVALUATION OF STATIN THERAPY IN DIABETIC PATIENTS UNDERGOING PERCUTANEOUS CORONARY INTERVENTION}

doi:10.1136/hrt.2010.208967.521

Liu Yuyang, Zhou Yujie, Hua Shen, Yang Shiwei, Gao Fei, Wang Zhijian, Shi Dongmei, Li Yueping, Ge Hailong, Liu Xiaoli, Han Hongya. Department of Cardiology, Beijing Anzhen Hospital, Capital Medical University, Beijing, China

Objective The long-term effect of statin therapy in diabetic patients after percutaneous coronary intervention (PCI) is not well established. Accordingly, we sought to determine whether statin therapy initiated at the time of percutaneous coronary intervention reduces total and cardiac mortality among diabetic patients.

Methods We collected data from 569 consecutive patients who underwent PCI. We then compared all-cause and cardiac mortality rates in 249 patients with diabetes mellitus of whom $74(29.7 \%)$ were treated with statin at the time of PCI. To adjust the variables that would have been related to the decision regarding statin administration, multivariate Cox regression was carried out.

Results During follow-up (4.4 \pm 1.3 years), 23 patients died (including 12 who died of cardiac causes). The Multivariate analysis showed statin therapy to be significantly associated with reduced cardiac mortality (HR 0.39, 0.16-0.95; $\mathrm{p}=0.039$ ), but not with allcause mortality.

Conclusion Statin therapy was associated with a significantly reduced risk of cardiac mortality in patients with diabetes mellitus and coronary artery disease after PCI.

\section{e0522 DUAL ANTIPLATELET PLUS TIROFIBAN THERAPY HAVE A BENEFICIAL EFFECT ON ACUTE CORONARY SYNDROME IN DIABETIC PATIENTS UNDERGOING PERCUTANEOUS CORONARY INTERVENTION}

doi:10.1136/hrt.2010.208967.522

Shen Hua, Zhou Yujie, Liu Yuyang, Yang Shiwei, Gao Fei, Wang Zhijian, Shi Dongmei, Han Hongya, Ge Hailong, Liu Xiaoli. Department of Cardiology, Beijing Anzhen Hospital, Capital Medical University, Beijing, China

Background Diabetes is strongly associated with clopidogrel resistance, thrombosis and the development of coronary artery disease (CAD). Some trials suggest that inhibition of glycoprotein IIb/IIIa can improve the outcome of clopidogrel resistance in patients undergoing percutaneous coronary interventions (PCIs). However, the efficacy of small-molecule IIb/IIIa receptor inhibitors in acute coronary syndrome (ACS) patients with diabetes undergoing PCI has not been specifically investigated.

Methods We randomised consecutive ACS patients with diabetes undergoing PCI, to tirofiban or placebo groups along with double antiplatelet therapy. High-dose bolus $(20 \mathrm{mg} / \mathrm{kg}$ per $3 \mathrm{~min}$ ) of tirofiban was administered immediately before PCI followed by $8 \mathrm{~h}$ continuous infusion $(0.15 \mathrm{mg} / \mathrm{kg}$ per $\mathrm{min})$. Postprocedural myocardial necrosis was assessed prospectively by measurement of cardiac troponin I (cTnI) at 6 and $24 \mathrm{~h}$ after PCI. The primary end-points were post-PCI coronary flow estimated by corrected TIMI frame count and post-PCI myocardial infarction.

Result 138 patients entered the study (66 randomised to placebo and 72 randomised to tirofiban). Post-PCI corrected TIMI frame count was $9.2 \pm 3.6$ in tirofiban and $13.0 \pm 7.6$ in placebo groups $(p=0.03)$. The prevalence of post-PCI myocardial infarction was similar in the two groups (17 vs $26 \%, p=0.167$, respectively).

Conclusion Up-stream use of tirofiban in ACS patients with diabetes undergoing PCI, along with double antiplatelet therapy, was associated with a decreased risk of distal embolisation.

\section{e0523 RISK FACTORS OF ACUTE RADIAL ARTERY OCCLUSION FOLLOWING TRANSRADIAL PERCUTANEOUS CORONARY INTERVENTION IN SENILE PATIENTS}

doi:10.1136/hrt.2010.208967.523

Zhao Yingxin, Zhou Yujie, Shi Dongmei, Guo Yonghe, Chen Wanjun, Yang Qing Shi Dongmei, Wang Zhijian, Nie Bin, Yan Zhenxian, Gao Fei. Department of Cardiology, Beijing Anzhen Hospital, Capital Medical University, Beijing, China

Background Compare with transfemoral percutaneous coronary intervention, the access site complication is less with transradial percutaneous coronary intervention (TRI). The acute radial artery occlusion (Acute RAO) is the most common complication following TRI. Although the incidence of acute RAO is less $(0 \sim 10 \%)$ and acute RAO couldn't induce upper-extremity ischaemia with positive Allen test patients, it makes re-TRI procedures impossible. To elucidate the risk factors of acute RAO is the best way to prevent it occurring.

Methods A total of 1256 positive Allen test patients ( $\geq 60$ years old) who underwent TRI (during May, 2004 to May, 2009) were divided into two groups: normal group and RAO group, according to whether the patient without or with acute RAO. Risk factors of acute RAO were analysed by logistic regression model.

Results Acute RAO occurred in 28 patients (2.2\%). Univariate analysis showed, the smaller size of sheath used, the higher incidence of acute RAO occurred. As compared to the patients in normal group, there are more female and diabetes mellitus patients in RAO group. The dose of heparin used in the operational procedure in RAO group were significantly less than normal group (3826 \pm 523 IU vs $7425 \pm 980 \mathrm{IU}, \mathrm{p}<0.01)$. The post-procedure duration of high-pressure compression haemostasis were longer in RAO group than normal group (378.9 $\pm 35.4 \mathrm{~min}$ vs $264.7 \pm 43.2 \mathrm{~min}, \mathrm{p}=0.03$ ). Logistic regression analyses showed that the dosage of heparin used in the procedure (RR: $2.812,95 \%$ CI 1.116 to $6.732, p=0.016$ ), the size of sheath (risk tatio: $4.978,95 \%$ CI 3.211 to $10.675, p=0.001$ ) and the post-procedure compression time (RR: 2.431, 95\% CI 1.389 to $5.010, p=0.034$ ) were independent risk factors for acute RAO.

Conclusion The incidence of acute RAO can be minimised by proper sheath selection, appropriate anticoagulation used during operational procedure, and avoiding prolonged duration of high-pressure compression haemostasis following the procedure.

\section{e0524 INTENSIVE CHOLESTEROL LOWERING WITH SIMVASTATIN IMPROVES THE OUTCOMES OF PERCUTANEOUS CORONARY INTERVENTION IN PATIENTS WITH ACUTE CORONARY SYNDROME}

doi:10.1136/hrt.2010.208967.524

Fu Xianghua, Jia Xinwei, Wang Yanbo, Wang Xuechao, Gu Xinshun, Zhang Jing, Hao Guozhen. The Second Hospital of Hebei Medical University, Shijiazhuang, Hebei, China

Objective To evaluate the immediate protective effects of intensive statin pretreatment on myocardial perfusion and myocardial ischaemic injury during PCI. 